\title{
甘油-水-氯化钠三元溶液中甘油浓度对甘油自扩散系数的影响
}

\author{
陈 聪* 李维仲宋永臣翁林岽张宁
}

(大连理工大学海洋能源利用与节能教育部重点实验室 大连 116024)

\begin{abstract}
摘要 利用分子动力学模拟方法研究了浓度对甘油-水-氯化钠三元溶液中甘油自扩散系数的影响. 随着甘油浓度的增 大，甘油的自扩散系数逐渐减小. 氢键分析表明，甘油自扩散系数的减小来源于其参与的甘油-水氢键数目的减少和甘 油-甘油氢键数目的增加.
\end{abstract}

关键词 分子动力学模拟; 自扩散系数; 甘油

\section{Effects of Glycerol Concentrations on Self-diffusion Coefficients of Glycerol in Glycerol-Water-Sodium Chloride Ternary Solutions}

\author{
Chen, Cong* Li, Weizhong Song, Yongchen Weng, Lindong Zhang, Ning \\ (Key Laboratory of Ocean Energy Utilization and Energy Conservation of Ministry of Education,
}

Dalian University of Technology, Dalian 116024)

\begin{abstract}
Glycerol self-diffusion coefficients in glycerol-water-sodium chloride ternary solutions with different concentrations have been predicted using molecular dynamics simulation method. As glycerol concentration increases, glycerol self-diffusion coefficient decreases. Hydrogen bonds analysis has been made and it has been found that decreasing of glycerol self-diffusion coefficients is caused by decreasing of mean number of glycerol-water hydrogen bonds and increasing of mean number of glycerol-glycerol hydrogen bonds.
\end{abstract}

Keywords molecular dynamics simulation; self diffusion coefficients; glycerol

甘油是一种常见物质，可以用作低温保护剂或生物 质原料, 因此, 获得了研究者的广泛关注.一些研究者 实验研究了甘油水溶液的热稳定性 ${ }^{[1]}$ 、粘度 ${ }^{[2 \sim 4]}$ 、水分活 度 $^{[5]}$ 、松弛动力 ${ }^{[6]}$ 、介质特性 ${ }^{[7]}$ 、过冷 ${ }^{[8]}$ 、冰晶生长 ${ }^{[9 \sim 12]}$ 和玻璃化行为 ${ }^{[13 \sim 16]}$. 一些研究者分析了甘油水溶液的 氢键特性，包括温度和浓度对氢键能力的影响 ${ }^{[17]}$ 、水分 子氢键的本质 ${ }^{[18]}$ 、氢键结构和动力 ${ }^{[19]}$ 以及氢键特性与甘 油的低温保护特性的联系 ${ }^{[20]}$. 与二元溶液相比, 甘油水-氯化钠三元溶液的研究相对较少. 有研究者研究了 甘油-水-氯化钠三元溶液的相图 ${ }^{[21 ~ 23]}$ 、动力粘度及水分
活度 ${ }^{[24]}$ 和氢键特性 ${ }^{[25,26]}$.

扩散系数是描述溶液动力学行为的一个重要参数. Tomlinson 利用 NMR 技术测量了不同温度下甘油的自 扩散系数 ${ }^{[27]}$. D'Errico 等 ${ }^{[28]}$ 测量了甘油水溶液中甘油和 水的扩散系数. 利用自由体积理论, $\mathrm{He}$ 等 ${ }^{[29}$ 拟合了甘油 水溶液中水自扩散系数的经验公式. Wang ${ }^{[30]}$ 研究了盐 离子对水自扩散系数的影响. Wang 等 ${ }^{[31]}$ 研究了氯化锂 对甲醇水溶液中水和甲醇自扩散系数的影响. 据作者所 掌握的文献，甘油-水一氯化钠三元溶液中甘油的自扩散 系数尚未见报道. 本文利用分子动力学模拟方法预测浓

*E-mail: congchen@dlut.edu.cn

Received July 15, 2011; accepted January 6, 2012.

Project supported by the Fundamental Research Funds for the Central Universities (No. DUT11NY01), Specialized Research Fund for the Doctoral Program of Higher Education (No. 20110041120035) and NSFC's Key Program Project (No. 50736001).

教育部中央高校基本科研业务费专项资金(No. DUT11NY01)，高等学校博士学科点专项科研基金新教师基金(No. 20110041120035)和国家自然科学 基金重点(No. 50736001)资助项目. 
度对甘油-水-氯化钠三元溶液中甘油自扩散系数的影 响, 并从氢键角度对浓度的影响进行分析.

\section{1 模拟细节和自扩散系数计算}

\section{1 模拟细节}

模拟采用 NAMD 程序 ${ }^{[32]}$, 水和甘油的势能模型分 别采用 $\mathrm{SPC} / \mathrm{E}^{[33]}$ 和 Reiling 建立的 CHARM22 格式的力 场 ${ }^{[34]}$. 初始速度采用 Gaussian 分布随机选取. 采用势能 截断法计算非键相互作用, 截断半径为 $1.35 \mathrm{~nm}$, 为保 证能量守恒, 势能从 $1.2 \mathrm{~nm}$ 缓慢减少到零. 在三个方向 上均采用周期性边界条件. 采用 Particle mesh Ewald 方

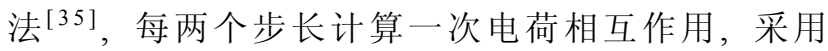
r-RESPA 方法 ${ }^{[36]}$ 求解体系运动方程, 采用 SHAKE 格 式 ${ }^{[37}$ 使水分子保持刚性. 模拟采用 NPT 系综, 时间步长 为 $2 \mathrm{fs}$, 采用 Nose-Hoover 方法 ${ }^{[38]}$ 将系统压力控制在 $101325 \mathrm{~Pa}$, 并用 Langevin 方法 ${ }^{[39]}$ 控制压力波动, 采用 Langevin 方法 ${ }^{\left[{ }^{[0]}\right]}$ 将系统温度控制在 $300 \mathrm{~K}$. 分别建立了 不同浓度的甘油-水-氯化钠三元体系, 如表 1 所示. 模 拟体系建立细节详见其它文献 ${ }^{[25,41]}$.

\section{表 1 模拟体系参数}

Table 1 Parameters of simulation boxes

\begin{tabular}{clllll}
\hline boxes & $n_{\mathrm{w}}$ & $n_{\mathrm{g}}$ & $n_{\text {ions }}$ & $x_{\mathrm{g}}$ & $x_{\text {ions }}$ \\
\hline bw & 0 & 0 & 768 & 0.00 & - \\
b1 & 2211 & 70 & 12 & 0.03 & 0.0052 \\
b2 & 1898 & 100 & 10 & 0.05 & 0.0050 \\
b3 & 1560 & 130 & 8 & 0.08 & 0.0047 \\
b4 & 1383 & 140 & 8 & 0.09 & 0.0052 \\
b5 & 1179 & 160 & 6 & 0.12 & 0.0045 \\
b6 & 1099 & 170 & 6 & 0.13 & 0.0047 \\
\hline
\end{tabular}

${ }^{a} n_{\mathrm{w}}, n_{\mathrm{g}}$ and $n_{\text {ions }}$ are the number of water molecules, glycerol molecules and ions (sodium cation and chlorine anion), respectively. $x_{\mathrm{g}}$ and $x_{\text {ions }}$ are the mole fractions of glycerol and ions, respectively.

\section{2 自扩散系数计算}

自扩散系数可以用速度自相关函数(Velocity Autocorrelation Function, VACF) 或者均方根位移 (Mean Square Displacement, MSD)来表示. 本文利用均方根位 移来计算自扩散系数. 均方根位移可以表示为:

$$
\operatorname{MSD}=\left\langle\sum_{j=1}^{N_{\mathrm{m}}}\left[r_{j}(t)-r_{j}(0)\right]^{2}\right\rangle
$$

而自扩散系数则可以表示为:

$$
D=\lim _{t \rightarrow \infty} \frac{1}{6 N_{\mathrm{m}} t} \text { MSD }
$$

其中, $D$ 为扩散系数, $N_{\mathrm{m}}$ 为总的原子数目, $t$ 为时间, $r_{j}(t)$
为原子 $j$ 在时刻 $t$ 真实的位移矢量. 本文在模拟时, 3 个 方向上均采用了周期性边界, 因此, 路径文件获得的位 移并不是原子的真实位移. 在本文模拟条件下，原子一 个时间步长内的位移远小于模拟体系的尺寸，因此，原 子的真实位移可以采用下式计算:

$$
r_{j}(t)=r_{j}(t)^{\prime}+\operatorname{nint}\left(\left[r_{j}(t-\Delta t)-r_{j}(t)^{\prime}\right] / L\right) L
$$

其中, $r_{j}(t)^{\prime}$ 为原子 $j$ 在时刻 $t$ 的 “虚拟” 位移(采用周期 性边界条件后的位移), $L$ 为体系尺寸, $\Delta t$ 为模拟时间步 长, $\operatorname{nint}(x)$ 表示不小于 $x$ 的最大整数.

\section{2 模拟结果}

甘油-水-氯化钠三元溶液中, 甘油分子的均方根位 移随模拟时长的变化如图 1 所示. 除了开始阶段, 均方 根位移随模拟时长呈线性变化. 利用均方根位移随模拟 时长的变化曲线可以求得甘油分子的自扩散系数, 结果 汇于表 2 .

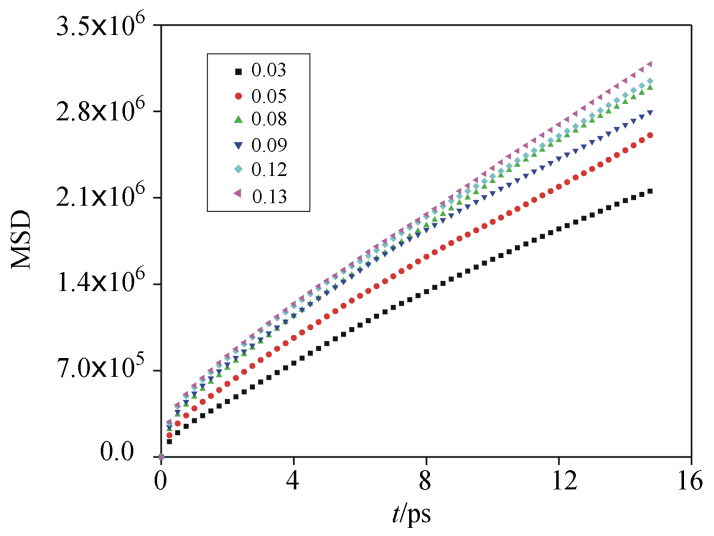

图 1 甘油-水-氯化钠三元溶液中, 甘油分子的均方根位移随 模拟时长的变化

Figure 1 Mean square displacements of glycerol molecules as a function of simulation time in glycerol-water-sodium chloride ternary solutions

表 2 甘油-水-氯化钠三元溶液中甘油的自扩散系数 ${ }^{a}$

Table 2 Diffusion coefficients of glycerol molecules in different simulation boxes

\begin{tabular}{lcll}
\hline$x_{\mathrm{g}}$ & $D /\left(10^{-10} \mathrm{~m}^{2} \cdot \mathrm{s}^{-1}\right)$ & $\begin{array}{l}\text { Standard error/ } \\
\left( \pm 10^{-10} \mathrm{~m}^{2} \cdot \mathrm{s}^{-1}\right)\end{array}$ & $R$ \\
\hline 0.03 & 7.523 & 0.0121 & 0.998 \\
0.05 & 6.071 & 0.0075 & 0.999 \\
0.08 & 5.367 & 0.0064 & 0.999 \\
0.09 & 4.435 & 0.0086 & 0.998 \\
0.12 & 4.265 & 0.0044 & 0.999 \\
0.13 & 4.253 & 0.0022 & 0.999 \\
\hline
\end{tabular}

${ }^{a}$ Mean square displacements have been linearly fitted with simulation time $t$ when $t$ is larger than 2 ps. The standard errors and correlation coefficients $R$ arising from linear regression have also been listed. 
表 2 中获得的甘油的自扩散系数是当模拟时长大于 $2 \mathrm{ps}$ 时, 甘油分子的均方根位移与模拟时长线性拟合的 结果. 拟合的相关系数均不小于 0.998 , 表明了均方根位 移与模拟时长良好的线性关系. 本文还模拟了纯水溶液 的自扩散系数, 模拟结果 $\left(2.212 \times 10^{-9} \mathrm{~m}^{2} / \mathrm{s}\right)$ 与实验结果 $\left(2.272 \times 10^{-9} \mathrm{~m}^{2} / \mathrm{s}\right)^{[42]}$ 吻合较好. 甘油一水-氯化钠三元溶 液中甘油自扩散系数的实验数据尚未见报道, 因此本文 模拟结果无法与实验结果进行直接比较. 本文的主要目 的是研究浓度对甘油自扩散系数的影响, 并从溶液氢键 结构的角度来分析这种影响. 由本文模拟可以发现, 随 着浓度的增加, 甘油自扩散系数不断减小. 溶质自扩散 系数随溶液浓度的这种变化趋势与其他研究者的结果 一致 $[28,31]$.

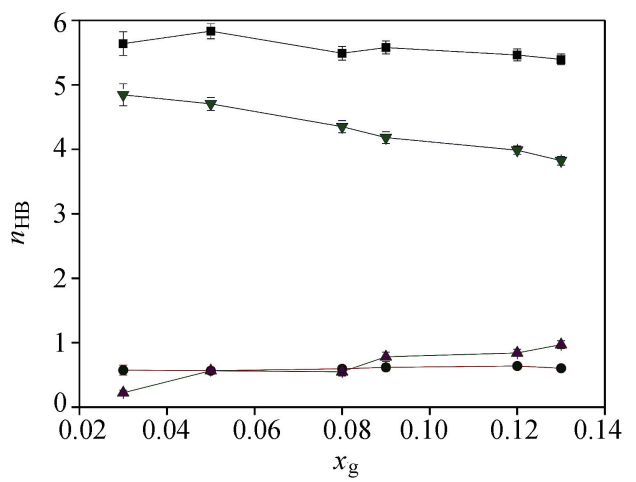

图 2 甘油-水-氯化钠三元溶液中, 平均每个甘油分子参与的 氢键数目随甘油浓度的变化 $(\mathbf{m}$ : 所有氢键; $\boldsymbol{\nabla}$ : 甘油一水之间氢 键; $\boldsymbol{\Delta}$ : 甘油-甘油分子间氢键; ・: 甘油分子内氢键)

Figure 2 The mean numbers of hydrogen bonds per glycerol molecule in glycerol-water-sodium chloride ternary solutions as a function of glycerol concentrations ( $\mathbf{m}$ : all hydrogen bonds; $\boldsymbol{\nabla}$ : hydrogen bonds between glycerol and water molecules; $\boldsymbol{\Delta}$ : hydrogen bonds between glycerol and glycerol molecules; $\bullet$ : intramolecular hydrogen bonds in glycerol molecules)

对于甘油-水-氯化钠三元溶液, 甘油分子参与的氢 键主要有三种: 甘油一水之间氢键, 甘油-甘油之间氢键 和甘油分子内氢键. 平均每个甘油分子参与的氢键数目 包括所有氢键以及不同种类的氢键随甘油浓度的变化 如图 2 所示. 虽然平均每个甘油分子参与的氢键数目随 浓度变化不大, 但是甘油分子参与的三种类型氢键的变 化趋势却有很大差异. 平均每个甘油分子参与的甘油水之间氢键随浓度的增大而减少. 当甘油摩尔浓度为 0.03 时, 平均每个甘油分子参与 4.845 个甘油-水氢键, 而当甘油摩尔浓度增大到 0.13 时, 平均每个甘油分子参 与 3.825 个甘油-水氢键, 减少了 $21 \%$. 而平均每个甘油 分子参与的甘油-甘油之间氢键则随浓度的增大而增大. 当甘油摩尔浓度为 0.03 时, 平均每个甘油分子参与 0.222 个甘油-甘油氢键，而当甘油摩尔浓度增大到 0.13
时，平均每个甘油分子参与 0.969 个甘油-甘油氢键，增 大了近 3.4 倍. 随甘油浓度增加, 平均每个甘油分子内 氢键数目几乎没有发生变化.

甘油自扩散系数随浓度的变化趋势可以通过平均 每个甘油分子参与的甘油-水氢键和甘油-甘油氢键数 目随浓度的变化趋势来解释. 水分子的自扩散系数在 $10^{-9} \mathrm{~m}^{2} / \mathrm{s}$ 量级, 而甘油分子的自扩散系数在 $10^{-10} \mathrm{~m}^{2} / \mathrm{s}$ 量级, 甘油一水氢键相互作用会使速度较慢的甘油分子 自扩散速度加快, 而使水分子自扩散速度变慢 ${ }^{[30,31]}$. 而 甘油一甘油之间的氢键作用则使得甘油分子的自扩散速 度变慢. 随甘油浓度的增大，平均每个甘油分子受到的 水分子的氢键作用不断减弱而其受到的甘油分子之间 的氢键作用不断增强，从而使得甘油分子的自扩散系数 不断减小. 在本文研究的甘油一水一氯化钠三元溶液中, $\mathrm{Na}^{+}$和 $\mathrm{Cl}^{-}$都水合一定量的水分子，同时包括部分来自 于甘油分子中的 $\mathrm{O}$ 和 $\mathrm{H}$ 原子 ${ }^{[25]}$. 除了存在水合作用, 离 子还能够扭曲水分子结构, 使得在某些离子溶液中随离 子浓度增大水的自扩散系数增大 ${ }^{[30]}$. 本文主要研究氢键 结构对甘油自扩散系数的影响, 对于离子的影响不再过 多讨论.

\section{3 结论}

利用分子动力学模拟方法研究了不同浓度的甘油水一氯化钠三元溶液中甘油的自扩散系数. 研究结果表 明, 随着甘油浓度的增大，甘油的自扩散系数逐渐减小. 当甘油摩尔浓度从 0.03 增大到 0.13 时，甘油分子的自扩 散系数从 $7.523 \times 10^{-10} \mathrm{~m}^{2} / \mathrm{s}$ 减小到 $4.253 \times 10^{-10} \mathrm{~m}^{2} / \mathrm{s}$. 氢键结构分析表明, 平均每个甘油分子参与的分子内氢 键数目随浓度变化不大, 而平均每个甘油分子参与的分 子间氢键数目随浓度发生显著变化. 平均每个甘油分子 参与的甘油-水氢键数目随浓度的增大而减小, 而平均 每个甘油分子参与的甘油-甘油氢键数目随浓度的增大 而增大. 甘油一水相互作用对甘油自扩散有加速作用, 而甘油一甘油相互作用对甘油自扩散有抑制作用. 综合 来看，随甘油浓度增大，甘油一水相互作用的加速作用 逐渐减弱而甘油一甘油相互作用的抑制作用逐渐增强, 从而甘油的自扩散系数逐渐减小.

致谢 模拟计算均在大连理工大学能源与动力学院高 性能计算服务器上进行，在此表示感谢.

\section{References}

1 Castello, M. L.; Dweck, J.; Aranda, D. A. G. J. Therm. Anal. Calorim. 2009, 97, 627. 
2 Morris, G. J.; Goodrich, M.; Acton, E.; Fonseca, F. Cryobiology 2006, 52, 323.

3 Gonzalez, J. A. T.; Longinotti, M. P.; Corti, H. R. J. Chem. Eng. Data 2011, 56, 1397.

4 Fita, P.; Punzi, A.; Vauthey, E. J. Phys. Chem. C 2009, 113, 20705.

5 Soujanya, J.; Satyavathi, B.; Prasad, T. E. V. J. Chem. Thermodyn. 2010, 42, 621.

6 Hayashi, Y.; Puzenko, A.; Feldman, Y. J. Non-Cryst. Solids 2006, 352, 4696.

7 Behrends, R.; Fuchs, K.; Kaatze, U. J. Chem. Phys. 2006, $124,144512$.

8 Sudo, S.; Shimomura, M.; Shinyashiki, N.; Yagihara, S. J. Non-Cryst. Solids 2002, 307-310, 356.

9 Murthy, S. S. N. J. Phys. Chem. B 2000, 104, 6955.

10 Hey, J. M.; MacFarlane, D. R. Cryobiology 1998, 37, 119.

11 Hey, J. M.; Macfarlane, D. R. Cryobiology 1996, 33, 205.

12 Vigier, G.; Vassoille, R. Cryobiology 1987, 24, 345.

13 Li, D. X.; Liu, B. L.; Liu, Y. S.; Chen, C. L. Cryobiology 2008, 56, 114.

14 Inaba, A.; Andersson, O. Thermochim. Acta 2007, 461, 44.

15 Zelent, B.; Nucci, N. V.; Vanderkooi, J. M. J. Phys. Chem. A 2004, 108, 11141.

16 Wang, B. C.; Li, D. X.; Liu, B. L.; Chen, C. L. Mol. Simul. 2010, 36, 1025.

17 Weng, L. D.; Chen, C.; Zuo, J. G.; Li, W. Z. J. Phys. Chem. A 2011, 115, 4729.

18 Kataoka, Y.; Kitadai, N.; Hisatomi, O.; Nakashima, S. Appl. Spectrosc. 2011, 65, 436 .

19 Chen, C.; Li, W. Z.; Song, Y. C.; Yang, J. J. Mol. Liq. 2009, 146, 23.

20 Dashnau, J. L.; Nucci, N. V.; Sharp, K. A.; Vanderkooi, J. M. J. Phys. Chem. B 2006, 110, 13670.

21 Shepard, M. L.; Goldston, C. S.; Cocks, F. H. Cryobiology 1976, 13, 9.

22 Jochem, M.; Korber, C. Cryobiology 1987, 24, 513.
23 Kleinhans, F. W.; Mazur, P. Cryobiology 2007, 54, 212.

24 Chenlo, F.; Moreira, R.; Pereira, G.; Bello, B. Eur. Food Res. Technol. 2004, 219, 403.

25 Chen, C.; Li, W. Z.; Song, Y. C.; Yang, J. THEOCHEM J. Mol. Struct. 2009, 916, 37.

26 Chen, C.; Li, W.; Song, Y.; Yang, J. Mol. Phys. 2009, 107, 673.

27 Tomlinson, D. J. Mol. Phys. 1973, 25, 735.

28 D'Errico, G.; Ortona, O.; Capuano, F.; Vitagliano, V. J. Chem. Eng. Data 2004, 49, 1665.

29 He, X.; Fowler, A.; Toner, M. J. Appl. Phys. 2006, 100, 074702 .

30 Wang, J. H. J. Phys. Chem. 1954, 58, 686.

31 Wang, P.; Anderko, A. Ind. Eng. Chem. Res. 2003, 42, 3495 .

32 Phillips, J. C.; Braun, R.; Wang, W.; Gumbar, J.; Tajkhorshid, E.; Villa, E.; Chipot, C.; Skeel, R. D.; Kale, L.; Schulten, K. J. Comput. Chem. 2005, 26, 1781.

33 Berendsen, H. J. C.; Grigera, J. R.; Straatsma, T. P. J. Phys. Chem. 1987, 91, 6269.

34 Reiling, S.; Schlenkrich, M.; Brickmann, J. J. Comput. Chem. 1996, 17, 450.

35 Darden, T.; York, D.; Pedersen, L. J. Chem. Phys. 1993, 98, 10089.

36 Procacci, P.; Marchi, M. J. Chem. Phys. 1996, 104, 3003.

37 Ryckaert, J. P. Mol. Phys. 1985, 55, 549.

38 Martyna, G. J.; Tobias, D. J.; Klein, M. L. J. Chem. Phys. 1994, 101, 4177.

39 Feller, S. E.; Zhang, Y.; Pastor, R. W.; Brooks, B. R. J. Chem. Phys. 1995, 103, 4613.

40 Brunger, A. T. X-PLOR, 3.1 ed., The Howard Higher Medical Institute and Department of Molecular Biophysics and Biochemistry, Yale University, 1992.

41 Chen, C.; Li, W.-Z. Acta Phys.-Chim. Sin. 2009, 25, 507.

42 Eisenberg, D.; Kauzmann, W. The Structure and Properties of Water, Oxford University Press, London, 1969. 